\title{
Effect of Shear Deformation on Texture Evolution in Rolled Pure Titanium
}

\author{
Huanping Yang and Yaomian Wang \\ School of Metallurgical Engineering, Xi' an University of Architecture and Technology, Xi' an 710055, China
}

\begin{abstract}
The cold rolling texture evolution under different strain states caused by coupled effects from different combinations of roll gap geometry and friction in pure titanium with initial typical recrystallized texture has been studied using viscoplastic self-consistent simulations. Under plane strain state, the texture is dominated by a typical cold rolling fiber texture $\mathrm{RD} / /<10 \overline{1} 0>$ in pure titanium. However, when shear deformation is induced and increased, $\mathrm{RD} / /<10 \overline{1} 0>$ fiber texture is decreased, whereas basal texture (0001)[1010] is increased and a strong partial fiber texture $\mathrm{RD} / /<2 \overline{1} \overline{10}>$ is formed. The variation of cold rolling texture at different strain states can be ascribed to variation of relative contribution from activation of prismatic, basal and pyramidal slip.
\end{abstract}

\section{Introduction}

Pure titanium is widely used in many key fields such as aerospace, chemical, energy and ocean industries due to its high specific strength and excellent corrosion resistance [1]. However, because of the inherent low symmetry of the hexagonal close packed (HCP) structure, pronounced mechanical anisotropy is occurred in pure titanium during plastic deformation, which will significantly influence subsequent further forming of these metals into different shapes/parts [2]. The mechanical anisotropy can be decreased by controlling texture through plastic deformation [3]. As known, the deformed texture can be largely determined by strain state. And extensive researches have been conducted to reveal the relationship between strain state and deformation texture in FCC and BCC metals and showed that deformation texture was strongly influenced by shear deformation [4-6]. As for HCP metals, few researches have also reported that inducing shear deformation can change deformation texture [7-8]. However, studies on relationship between shear deformation and texture evolution in pure titanium are rarely reported.

Therefore, in the present work, the effect of the strain state focused on shear deformation caused by coupled effects of roll gap geometry and friction on rolling texture evolution in pure titanium was investigated using crystal plasticity simulation.

\section{Simulation methods and parameters}

The rolling texture under different strain states was simulated by means of viscoplastic self-consistent(VPSC) code. The detailed VPSC model and the predominant twin reorientation scheme are described in the work of
Tomé [9] and Lebensohn et al.[10]. The Voce law, shown by Equation 1, is used to describe hardening behavior on different slip and twin systems.

$$
\begin{gathered}
\tau^{s}(\Gamma)=\tau_{0}+\left(\tau_{1}+\theta_{1} \Gamma\right)\left[1-\exp \left(\frac{-\theta_{0} \Gamma}{\tau_{1}}\right)\right] \\
\Gamma=\int_{0}^{t} \sum_{s}\left|\gamma^{s}\right| d t
\end{gathered}
$$

where $\Gamma$ expressed in Equation 2 being the accumulated shear within time $t$ on all slip systems, and $\left|\gamma^{s}\right|$ is the shear rate on slip system $s$. In the simulation, the prismatic $<\mathrm{a}>\operatorname{slip}\{10 \overline{1} 0\}<1 \overline{2} 10>$, the basal $<\mathrm{a}>$ slip $\{0001\}<1210>$, the first-order pyramidal $<\mathrm{c}+\mathrm{a}>$ slip $\{101$ $1\}<\overline{1123}>$ and two twinning modes, namely the tensile twinning $\{10 \overline{1} 2\}<\overline{1011}>$ and compressive twinning $\{11 \overline{2}$ $2\}<1123>$, were taken into account. $\tau_{0}, \tau_{1}, \theta_{0}$ and $\theta_{1}$ are hardening parameters for each deformation system. The hardening behaviors represented by four parameters of the five deformation modes that lead to the best texture predictions are illustrated in Figure 1.

The different strain states were obtained by changing combinations of roll gap geometry and friction. As for rolling deformation, it is customary to assume plane strain state $\varepsilon_{11}=-\varepsilon_{33}$ and all other components $\varepsilon_{22}, \varepsilon_{12}, \varepsilon_{13}$, $\varepsilon_{23}$ are imposed to be zero. However, strong deviations from the plane strain prevail due to geometrical changes within the roll gap and the friction between roll surface and sheet surface, which leads to nonzero displacement gradient component $e_{13}$ and $e_{31}$, resulting nonzero component $\varepsilon_{13}$. The different strain states are described by a ratio $\varepsilon_{13} / \varepsilon_{11}$ in the work. The shear deformation from 
geometrical and frictional effects is simplified as the material goes into and out the roll gap and is shown in Figure 2(a) and Figure 2(b), respectively. The shear deformation caused by roll gap geometry and friction is denoted as $\gamma_{g}$ and $\gamma_{f}$, which is described by the ratio of shear $e_{13}$ to $\mathrm{e}_{11}$ and $e_{31}$ to $\mathrm{e}_{11}$, respectively. According to Lee et al. [5], when shear deformation is caused just by roll gap geometry or friction, texture is nearly identical when $\gamma_{g}=\gamma_{f}$. And the geometrical effects and frictional effects can cancel each other. Therefore, the combined effects of roll gap geometry and friction are considered, and the deformation gradient is described by Equation 3 .

$$
E_{\text {in }}=\left|\begin{array}{ccc}
0.5 & 0 & -\gamma_{g} \\
0 & 0 & 0 \\
\gamma_{f} & 0 & -0.5
\end{array}\right| e_{11} \quad E_{\text {out }}=\left|\begin{array}{ccc}
0.5 & 0 & -\gamma_{g} \\
0 & 0 & 0 \\
\gamma_{f} & 0 & -0.5
\end{array}\right| e_{11}
$$

Four different state states from coupled effects of roll gap geometry and friction are listed in Table 1, i.e., $\varepsilon_{13} / \varepsilon_{11}=0$, $\varepsilon_{13} / \varepsilon_{11}=0.3, \varepsilon_{13} / \varepsilon_{11}=0.5$ and $\varepsilon_{13} / \varepsilon_{1 I}=1$. A true strain of 0.07 per pass has been imparted for all the samples to a total true strain of 0.7 , corresponding to rolling reduction of $50 \%$.

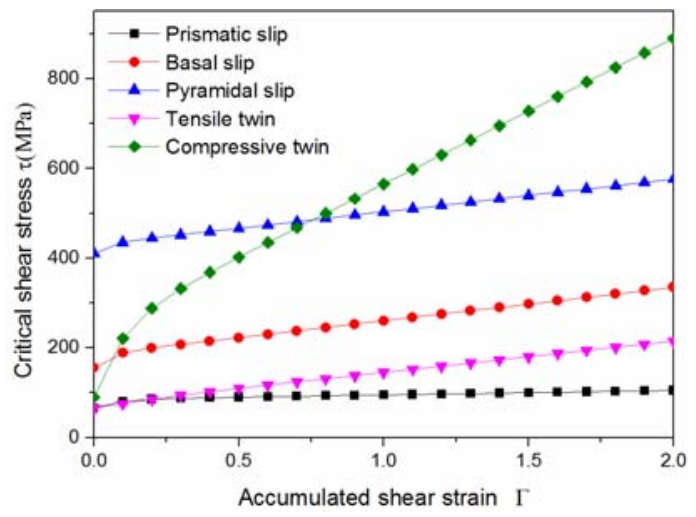

Fig. 1. Hardening behaviors of the slip/twin systems.
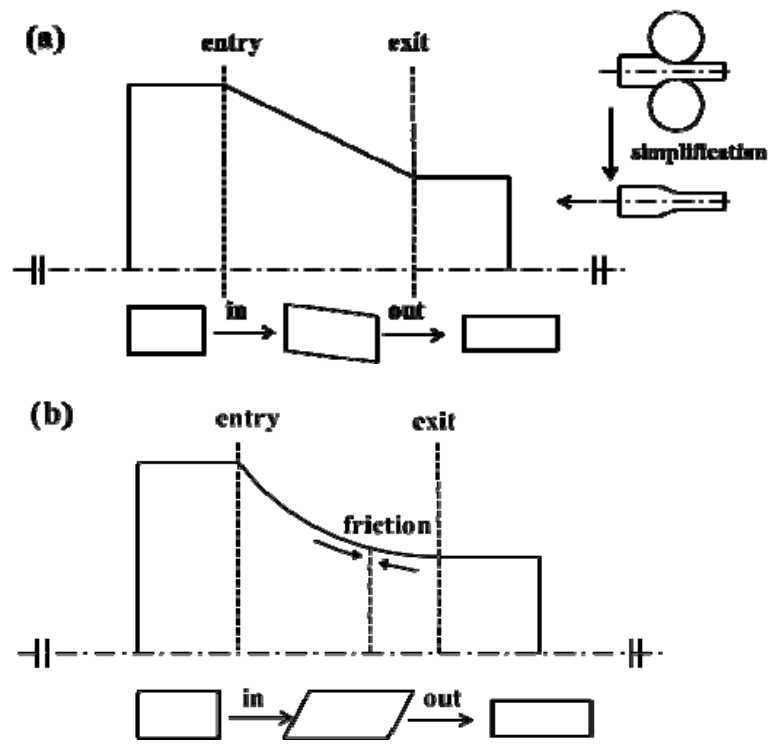

Fig. 2. Schematic diagram showing shear strain induced by roll gap geometry(a) and friction(b).
The texture evolution was characterized by the orientation distribution function (ODF) in the Euler space of $\left(\varphi_{1}, \phi, \varphi_{2}\right)$ following Bunge convention. When describing a specific orientation, the relationship between crystal and sample coordinate system is defined as $\mathrm{RD}=[\overline{1}$

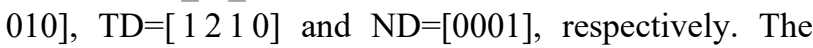
texture was calculated using MTEX toolbox [11] by imposing the orthorhombic sample symmetry for rolling process.

Table 1. Different strain states from different combinations of roll gap geometry and friction.

\begin{tabular}{ccc}
\hline$\varepsilon_{13} / \varepsilon_{11}$ & $\gamma_{\mathrm{g}}$ & $\gamma_{\mathrm{f}}$ \\
\hline 0 & 1 & 1 \\
0.3 & 0.7 & 1 \\
0.5 & 0.5 & 1 \\
1 & 0 & 1 \\
\hline
\end{tabular}

\section{Results and Discussion}

The initial texture is shown in Figure 3(a), which is a typical texture commonly observed in recrystallized pure titanium [12] and can be characterized by orientation range extended between the orientation $\mathrm{B}=\{0001\}<10 \overline{1}$ $0> \pm 40^{\circ}$ and $\mathrm{E}=\{0001\}<11 \overline{20}> \pm 40^{\circ}$ with peak intensity at component $\mathrm{E}$. When the pure titanium is cold rolled by $50 \%$ at plane strain state, i.e., $\varepsilon_{1 I}=-\varepsilon_{33}, \gamma_{g}=\gamma_{f}=0$, the texture is shown in Figure 3(b). In order to compare conveniently, Figure 3(c) gives the common orientations and fibers in cold rolled pure titanium. As shown, the main texture under plane strain state can be characterized by a typical cold rolling texture, i.e., $\mathrm{RD} / /<10 \overline{10}>$ fiber texture, which verifies the reliability of the set of hardening parameters. And the $\mathrm{RD} / /<10 \overline{10}>$ fiber texture is extending from component $\mathrm{A}$ (0001) $\left[\begin{array}{lll}10 & \overline{1} & 0\end{array}\right]$ to component $\mathrm{C}(\overline{12} \overline{10})[10 \overline{10}$ ] with maximum intensity near component B but shifting toward little $\phi$ at $(\overline{12} \overline{15})$ [1010], which is a dominant texture commonly found in cold rolled pure titanium [13].

Figure 4 shows the texture in pure titanium cold rolled by $50 \%$ at different strain states. It can be seen that under coupled effects of roll gap geometry and friction and $\gamma_{g}=\gamma_{f}, \varepsilon_{13} / \varepsilon_{11}$ is equal to 0 because of just balanced effects, and the texture is similar to plane strain rolling texture shown in Figure 3(b). The results are in good agreement with that in face-centered cubic metals calculated by a fully constrained Taylor model, i.e., the simulated texture is identical to the plane strain simulation when $\gamma_{g}=\gamma_{f}$ [5]. When $\varepsilon_{13} / \varepsilon_{11}$ is increased to 0.3 due to unbalanced effects of roll gap geometry and friction, the texture characteristics have not changed much. When $\varepsilon_{13} / \varepsilon_{11}$ is further increased to 0.5 , the texture is obviously changed.

The dominant plane strain texture $\mathrm{RD} / /<10 \overline{1} 0>$ fiber texture is significantly decreased, while the basal texture (0001)[1010] is remarkably increased. The phenomenon that the basal texture is strengthened with increased shear 
deformation from VPSC simulation agrees well with the experimental results of Milner et al., who reported a $\pm 30^{\circ}$ transverse direction split basal texture was evolved to primarily basal texture when inducing shear deformation by isolated shear rolling [7]. And when $\varepsilon_{13} / \varepsilon_{11}$ is increased up to 1 , the $\mathrm{RD} / /<1010>$ fiber texture is further decreased and the basal fiber texture with maximum intensity at (0001)[10 10$]$ is further increased. Also, another partial fiber texture $\mathrm{RD} / /<2 \overline{1} 10>$ with peak intensity close to component $\mathrm{E}$ but shifting to orientation

$(01 \overline{13})[2110]$ is formed, which becomes the strongest component.
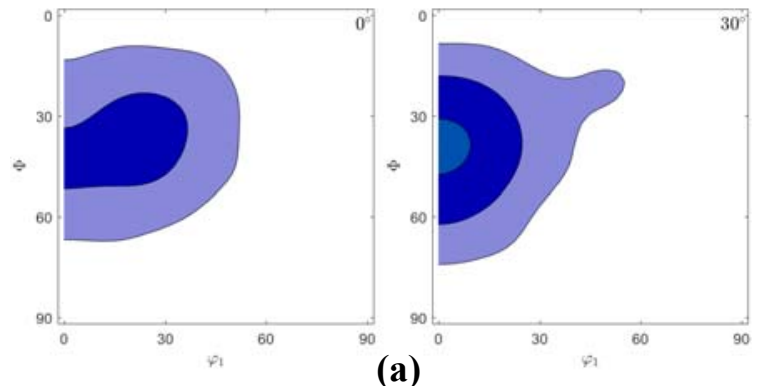

(a)
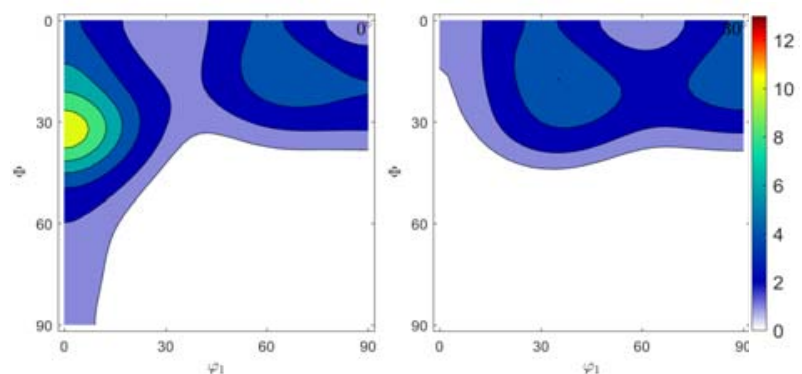

(b)
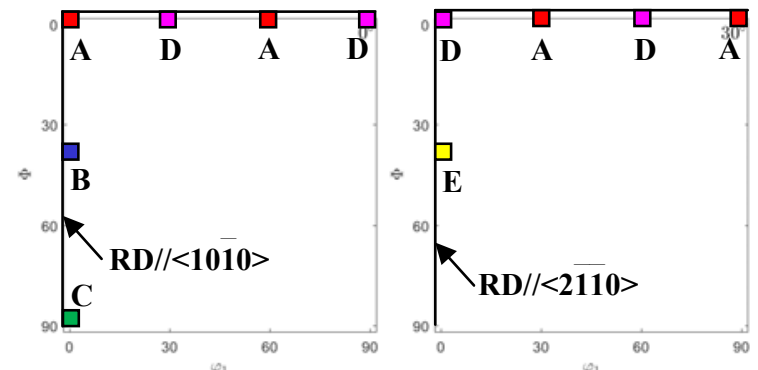

(c)

Fig. 3. Constant $\varphi_{2}=0^{\circ}$ and $\varphi_{2}=30^{\circ}$ of the ODF in initial pure titanium(a), cold rolled pure titanium under plane strain state(b) and common rolled texture components and fibers in pure titanium(c).
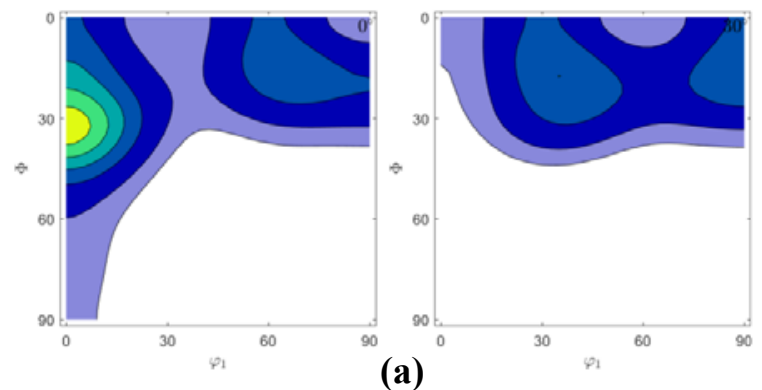

(a)
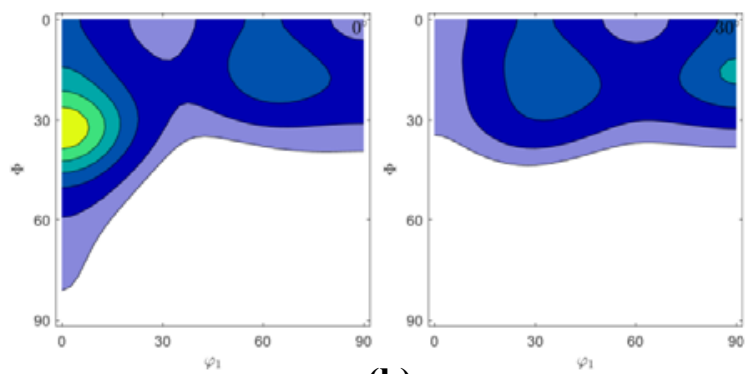

(b)
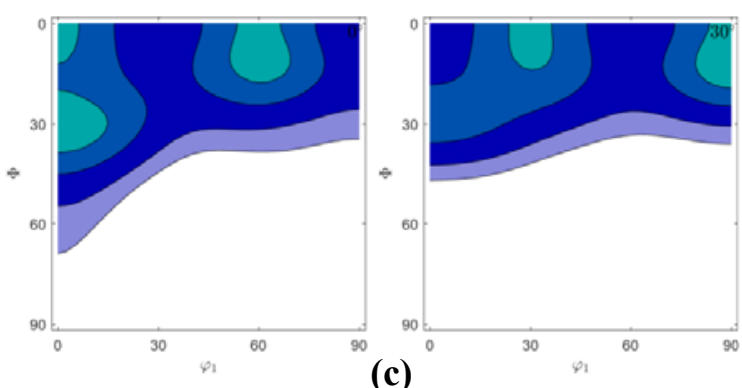

(c)
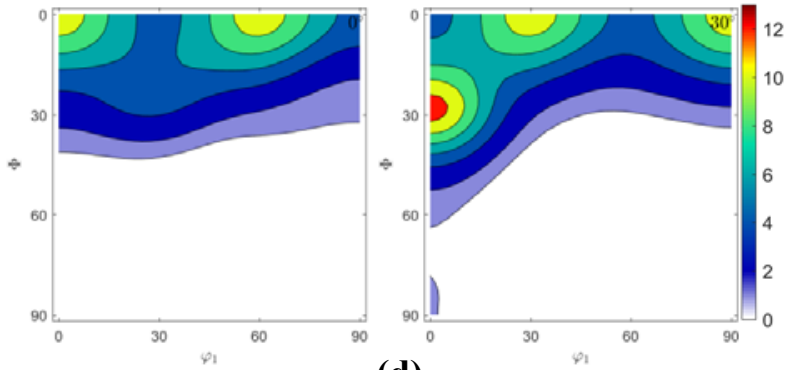

(d)

Fig. 4. Constant $\varphi_{2}=0^{\circ}$ and $\varphi_{2}=30^{\circ}$ of the ODF in cold rolled pure titanium at different strain states: (a) $\varepsilon_{13} / \varepsilon_{11}=0$, (b) $\varepsilon_{13} / \varepsilon_{11}=0.3$, (c) $\varepsilon_{13} / \varepsilon_{11}=0.5$ and (d) $\varepsilon_{13} / \varepsilon_{11}=1$.

The difference in cold rolled texture of pure titanium under different strain states is closely related to slip/twin activities. Figure 5 gives the slip/twin activities obtained from VPSC simulations. It shows that prismatic slip is the first dominant slip system under plane strain state, which contributes significantly to the formation of dominated $\mathrm{RD} / /<10 \overline{10}>$ fiber texture. And basal slip is the secondary dominant slip system. Also, the activation fraction of the pyramidal slip and two twinning systems are relatively lower. When $\varepsilon_{13} / \varepsilon_{11}$ is increased to 0.3 , the slip/twin activities have not changed much, which is consistent with the texture evolution shown in Figure 4(b). And when $\varepsilon_{13} / \varepsilon_{11}$ is increased to 0.5 and 1 , the activation of slip/twin systems varies significantly. The activation of prismatic slip is decreased, whereas the activation of the basal and pyramidal slip is increased. The larger the $\varepsilon_{13} / \varepsilon_{11}$ is, the more the activation fraction of the prismatic slip, basal and pyramidal slip varies. The dominated near E component caused by pyramidal slip have also found in cold rolled submicron grain size pure titanium [14]. Certainly, because the texture evolution may be influenced by rolling reduction per pass, which is a true strain of 0.07 used in the paper, further researches should be conducted systematically. 

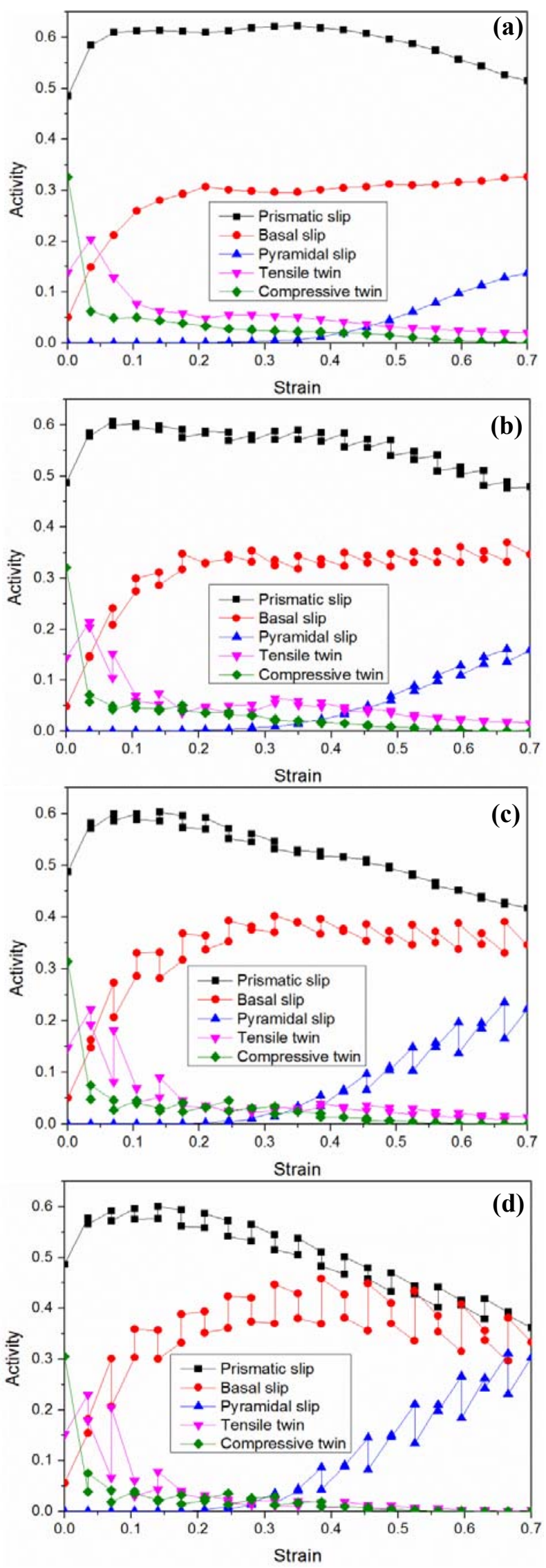

Fig. 5. Activity for slip/twin systems in cold rolled pure titanium at different strain states: (a) $\varepsilon_{13} / \varepsilon_{11}=0$, (b) $\varepsilon_{13} / \varepsilon_{11}=0.3$, (c) $\varepsilon_{13} / \varepsilon_{11}=0.5$ and (d) $\varepsilon_{13} / \varepsilon_{11}=1$.

\section{Conclusions}

(1) Strain state significantly influences cold rolling texture evolution in pure titanium. Under plane strain state, the texture is dominated by a typical fiber texture

$\mathrm{RD} / /<1010>$ with maximum intensity at $(1215)[1010]$. When shear deformation is induced by coupled effects of roll gap geometry and friction and as the shear deformation is increased, the $\mathrm{RD} / /<1010>$ fiber texture is decreased, whereas the basal texture (0001)[10 10 ll 0 is increased and a strong partial fiber texture $\mathrm{RD} / /<2110>$ with peak intensity at $(01 \overline{13})[2 \overline{11} 0]$ is formed.

(2) The variation of cold rolling texture with increased shear deformation can be attributed to decreased activation of prismatic slip and increased activation of basal and pyramidal slip compared with that under plane strain state.

\section{References}

1. G. Lütjering, J.C. Williams, Titanium, second ed., Springer-Verlag, Berlin, 2007

2. H. Inagaki, Z Metallkd. 83, pp40-46, (1992)

3. Z.S. Zhu, R.Y. Liu, M.G. Yan, C.X. Cao, J. Mater. Sci. 32, pp5163-5167, (1997)

4. O. Engler, C.N. Tomé, M.Y. Huh, Metall. Mater. Trans. A 31, pp2299-2315, (2000)

5. C.S. Lee, B.J. Bunge, Metall. Trans. A 22, pp26372643, (1991)

6. K.H. Kim, D.N. Lee, Acta Mater. 49, pp2583-2595, (2001)

7. J.L. Milner, F. Abu-Farha, T. Kurfess, V.H. Hammond, Mater. Sci. Eng. A 619, pp12-25, (2014)

8. W.J. Kim, S.J. Yoo, H.T. Jeong, D.M. Kim, B.H. Choe, J.B. Lee, Scripta Mater. 64, pp49-52, (2011)

9. C.N. Tomé, R.A. Lebensohn, U.F. Kocks, Acta Metall. Mater. 39, pp2667-2680, (1991)

10. R.A. Lebensohn, C.N. Tomé, Acta Metall. Mater. 41, pp2611-2624, (1993)

11. F. Bachmann, R. Hielscher, H. Schaeben, Solid State Phenom. 160, pp63-68, (2010)

12. N. Bozzolo, N. Dewobroto, T. Grosdidier, F. Wagner, Mater. Sci. Eng. A 397, pp346-355, (2005)

13. H.P. Lee, C. Esling, H.J. Bunge, Texture. Microstruct. 7, pp317-337, (1988)

14. N.P. Gurao, S. Suwas, J. Mater. Res. 26, pp523-532, (2011) 